\title{
Quantum Spectrometry for Arbitrary Noise
}

\author{
Daniel Goldwater and P. F. Barker \\ Department of Physics and Astronomy, University College London, Gower Street, London WC1E 6BT, United Kingdom \\ Angelo Bassi \\ Department of Physics, University of Trieste, Strada Costiera 11, 34151 Trieste, Italy \\ and Istituto Nazionale di Fisica Nucleare, Trieste Section, Via Valerio 2, 34127 Trieste, Italy \\ Sandro Donadi \\ Frankfurt Institute for Advanced Studies (FIAS), Ruth-Moufang-Straße 1, 60438 Frankfurt am Main, Germany
}

(Received 1 February 2019; published 2 December 2019)

\begin{abstract}
We present a technique for recovering the spectrum of a non-Markovian bosonic bath and/or nonMarkovian noises coupled to a harmonic oscillator. The treatment is valid under the conditions that the environment is large and hot compared to the oscillator, and that its temporal autocorrelation functions are symmetric with respect to time translation and reflection — criteria which we consider fairly minimal. We model a demonstration of the technique as deployed in the experimental scenario of a nanosphere levitated in a Paul trap, and show that it would effectively probe the spectrum of an electric field noise source from $10^{2}$ to $10^{6} \mathrm{~Hz}$ with a resolution inversely proportional to the measurement time. This technique may be deployed in quantum sensing, metrology, computing, and in experimental probes of foundational questions.
\end{abstract}

DOI: 10.1103/PhysRevLett.123.230801

Introduction.-Noise is an unavoidable feature of all physical measurements, and often their main impediment. The study of noise in quantum physics is a field in its own right $[1,2]$, and the peculiar structure of the noise spectrum is of central importance to quantum metrology [3] and more generally to the theory of open quantum systems [4]. However, in the majority of treatments, the exact nature of the noise spectrum is left as an unknown quantity-if it is not idealized as Markovian, it will typically be ascribed a simple structure, such as Ohmic [5], though a larger taxonomy exists [6].

In this Letter we introduce a scheme which is capable of measuring the spectrum of arbitrary noises or bosonic baths. In itself this constitutes a new quantum sensing tool comparable to other spectrometers [7-9], but it may also be used to improve existing techniques by helping metrological schemes which deal with noise [10]. It can be enacted on any harmonic oscillator which can be prepared in a cold initial state, and whose resonant frequency may be varied. Since the vast majority of quantum sensors, and indeed quantum experiments, are based around the behavior of harmonic oscillators, this implies a potentially wide range of applications.

One direct utilization which we will demonstrate in this work would be to study the structure of the electric field noise found in electric particle traps, which arises from the electrodes and is determined by several factors [11] in a way not yet fully understood $[12,13]$. To make this demonstration, we will model the specific scenario of an electrically levitated charged nanosphere; in such a setting, the environmental noise conditions are such that an accurate reconstruction of the electric field noise spectrum ought to be possible over a wide range-between $10^{2}$ and $10^{6} \mathrm{~Hz}$. Further, this ability to accurately characterize the environmental noise spectrum may find application in quantum computing, where such knowledge would enable the development of optimized dynamical decoupling protocols tailored to the specific environment of the qubit(s) [14]. It may also find applications in short-range force sensing $[15,16]$, where the spectrum characterizing the interaction between the force being studied and the behavior of the oscillator can be subjected to a similar treatment.

Here we make a proposal in three parts. First, we describe a mathematical formalism through which the spectrum of a general bosonic bath coupled to a quantum harmonic oscillator may be recovered through experiment (granted certain assumptions, and up to a degree of uncertainty). Second, we propose a specific experimental scenario which is particularly suited to this task (that of a levitated nanoparticle) and demonstrate its theoretical performance. Third, we examine an example case-that of an electric field noise with a nontrivial spectrum-and show how the experimental scenario of the previous section would be able to recover this spectrum through the suggested technique.

Formalism.-We begin by considering a Hamiltonian which describes a harmonic oscillator $\mathcal{S}$ coupled to a bosonic bath of independent harmonic oscillators $\mathcal{B}$ : 


$$
\hat{H}=\hat{H}_{\mathcal{S}}+\hat{H}_{\mathcal{B}}+\hat{H}_{\mathcal{I}},
$$

in which the three terms above represent the system, bath, and interaction Hamiltonians, respectively. Setting $\hbar=1$, they are given by

$$
\begin{aligned}
& \hat{H}_{\mathcal{S}}=\omega_{m}\left(\hat{a}^{\dagger} \hat{a}+\frac{1}{2}\right), \\
& \hat{H}_{\mathcal{B}}=\sum_{\alpha} \omega_{\alpha}\left(\hat{b}_{\alpha}^{\dagger} \hat{b}_{\alpha}+\frac{1}{2}\right), \\
& \hat{H}_{\mathcal{I}}=-\hat{q} \sum_{\alpha} g_{\alpha} \hat{q}_{\alpha},
\end{aligned}
$$

where $\omega_{m}$ represents the mechanical frequency of the oscillator and $\hat{a}^{\dagger}, \hat{a}, \hat{q}$ give its creation, annihilation, and position operators, respectively. Similarly, we decompose $\mathcal{B}$ into $\alpha$ modes, whose creation, annihilation, and position operators are $\hat{b}_{\alpha}^{\dagger}, \hat{b}_{\alpha}$, and $\hat{q}_{\alpha}$, respectively. The interaction between the system and the $\alpha$ mode of the bath is described through a position-position coupling whose strength is given by $g_{\alpha}=m_{\alpha} \omega_{\alpha}^{2}$ whose value can be settled by an appropriate choice of the bath oscillator masses.

The first description of the dynamics of this type of system was given by Caldeira and Leggett in their seminal paper [17], in which they derived a master equation for this scenario using the Born-Markov approximation, valid in the limit of high temperatures of the bath. This result was improved by Hu et al. [18], who derived a master equation which is exact, and valid for any temperature. The subject has more recently been studied in Refs. $[19,20]$, where the $\mathrm{Hu}$ et al. master equation was derived in the form

$$
\begin{aligned}
\frac{d}{d t} \rho_{t}= & -i\left[\hat{H}_{S}-\Xi(t) q^{2}, \rho_{t}\right]+\Gamma(t)\left[\hat{q},\left[\hat{q}, \rho_{t}\right]\right] \\
& +\Theta(t)\left[\hat{q},\left[\hat{p}, \rho_{t}\right]\right]+i \Upsilon(t)\left[\hat{q},\left\{\hat{p}, \rho_{t}\right\}\right],
\end{aligned}
$$

with $\rho_{t}$ the density matrix at time $t$, and $\hat{p}$ the momentum operator. It is Eq. (5) which we shall use as a jumping off point for developing our formalism - it is very general-for example, the bath need not be thermal for the equation to be valid. It must, however, begin in a Gaussian state. (For an example of a spectrometer which could probe non-Gaussian noise, see Refs. [21,22].) In Appendix 1 of the Supplemental Material we detail an alternative derivation [23].

The exact definitions of the time-dependent coefficients $\Xi(t), \Gamma(t), \Theta(t)$, and $\Upsilon(t)$ are given through recursive series expansions and can be found in Ref. [19]. However, in the limit of a weak coupling between the system and bath (a limit which we will now assume), one can safely make a first order approximation which greatly simplifies the expressions for these coefficients. Further to this, we will consider only the regime where the damping effects of the bath upon the system will be negligible compared to its heating effects, which is mathematically equivalent to assuming that the bath correlation function is real [19]. Note that, in the case of thermal baths, the assumption that dissipative effects are negligible is equivalent to assuming that the temperature of the bath is much higher than that of the system. We will go into further detail regarding these simplifications in Appendix 1 of the Supplemental Material.

Taking these simplifications, one gets $\Xi(t)=\Upsilon(t)=0$ and

$$
\begin{aligned}
& \Gamma(t)=-\int_{0}^{t} d s C(t, s) \cos \left[\omega_{m}(t-s)\right], \\
& \Theta(t)=\int_{0}^{t} d s C(t, s) \frac{\sin \left[\omega_{m}(t-s)\right]}{m \omega_{m}},
\end{aligned}
$$

in which $C(t, s)=\operatorname{Tr}\left[\hat{B}(t) \hat{B}(s) \rho_{B}\right]$ is the two-time correlation function for the bath operator $\hat{B}=\sum_{\alpha} g_{\alpha} \hat{q}_{\alpha}$. The Fourier transform of $C(t, s)$-the spectrum of the noise function in frequency space-is the object which our spectrometer will ultimately uncover through a study of its impact upon the system. Using these simplifications, Eq. (5) becomes

$$
\frac{d}{d t} \rho_{t}=-i\left[\hat{H}_{S}, \rho_{t}\right]+\Gamma(t)\left[\hat{q},\left[\hat{q}, \rho_{t}\right]\right]+\Theta(t)\left[\hat{q},\left[\hat{p}, \rho_{t}\right]\right] .
$$

For the spectrometer to function as a viable measurement instrument, we must of course select an observable to monitor. Here we show that the number operator $\hat{n}=\hat{a}^{\dagger} \hat{a}$ is ideal. It is experimentally straightforward to measure, and can be used to recover $C(t, s)$ unambiguously.

The equation of motion for the expected occupation number can be found via $(d / d t)\langle\hat{n}\rangle_{t}=\operatorname{Tr}\left[\hat{n} \mathcal{L} \rho_{t}\right]$, in which $\mathcal{L} \rho_{t}$ is a superoperator on $\rho_{t}$ which summarizes the righthand side of Eq. (8). Using this, the cyclicity of the trace, and a little algebra, we find that

$$
\frac{d}{d t}\langle\hat{n}\rangle_{t}=\frac{1}{2 m \omega_{m}} \int_{0}^{t} C(t, s) \cos \left[\omega_{m}(t-s)\right] .
$$

Now, if we assume that the correlation function is invariant with respect to both time reversal and time translation, i.e., that $C(t, s)=C(|t-s|)$, we can rewrite the right-hand side of Eq. (9) in a more convenient form by using the relation

$$
\int_{0}^{t} d s C(|s-t|) \cos \left[\omega_{m}(s-t)\right]=\frac{1}{2} \int_{-t}^{t} d y C(y) e^{i \omega_{m} y} .
$$

Introducing the Fourier expansion of $C(y)$,

$$
C(y)=\frac{1}{2 \pi} \int_{-\infty}^{\infty} d \nu \tilde{C}(\nu) e^{-i \nu y},
$$

Eq. (9) becomes 


$$
\frac{d}{d t}\langle\hat{n}\rangle_{t}=\frac{1}{4 \pi m \omega_{m}} \int_{-\infty}^{\infty} d \nu \tilde{C}(\nu) \frac{\sin \left[\left(\omega_{m}-\nu\right) t\right]}{\left(\omega_{m}-\nu\right)}
$$

which can be solved to get

$$
\langle\hat{n}\rangle_{t}=\langle\hat{n}\rangle_{0}+\frac{1}{2 \pi m \omega_{m}} \int_{-\infty}^{\infty} d \nu \tilde{C}(\nu) \frac{\sin ^{2}\left[\left(\omega_{m}-\nu\right) t / 2\right]}{\left(\omega_{m}-\nu\right)^{2}} .
$$

This equation contains the basic capabilities of the spectrometer, and as such it demands some examination. In the white noise limit $\tilde{C}(\nu)=D_{p}$ with $D_{p}$ being some positive constant, it gives the well-known behavior: $\langle\hat{n}\rangle_{t}=\langle\hat{n}\rangle_{0}+D_{p}^{\prime} t$, where $D_{p}^{\prime}=D_{p} / 4 m \omega_{m}$ quantifies the heating rate due to momentum diffusion. While the integral in Eq. (13) is of course unsolvable without knowing $\tilde{C}(\nu)$, its form allows us to estimate the function of interest. The function $\sin ^{2}(\theta t / 2) / \theta^{2}$ forms a peak around $\theta=0$ of width $4 \pi / t$. For us, this means that the envelope of the peak will serve analogously to a flared $\delta$ function, selecting the effects of $\tilde{C}(\nu)$ in the region of $\nu=\left[\omega_{m}-(2 \pi / t), \omega_{m}+(2 \pi / t)\right]$. We can deploy this approximation, in Eq. (13), replacing the integral with $\tilde{C}\left(\omega_{m}\right)(\pi t / 2)$ and rearranging to get

$$
\tilde{C}\left(\omega_{m}\right)=\frac{4 m \omega_{m}}{t}\left(\langle n\rangle_{t}-\langle n\rangle_{0}\right) .
$$

Note that although this is appears to be linear in $\omega_{m}$, the dominant term will typically be the $\langle n\rangle_{t}$ of Eq. (13), in which the coefficient in front of the integral is $\propto 1 / \omega_{m}$, neutralizing the linear scaling, and that $\langle n\rangle_{t}$ has a more complicated dependence upon $\omega_{m}$ in the integrand related to the noise spectrum. If $\omega_{m}$ is changeable, this will allow us to scan through the range of available frequencies and see how $\tilde{C}(\nu)$ behaves across the range with an error $\propto 1 / t$. This, then, forms the protocol for the spectrometer: to take measurements of $\langle\hat{n}\rangle_{t}$ at different values of $\omega_{m}$, and use the results to reconstruct $\tilde{C}(\nu)$ via Eq. (14). The accuracy of this reconstruction will be improved with an increasing time $t$ taken per measurement.

Equation (13) has the same structure as Eq. (18) from Ref. [24], which focuses on the decoherence of superconducting qubits resulting from their interaction with a classical noise. However, there are fundamental differences between the two approaches. First of all, the systems interacting with the noise considered in Ref. [24] are qubits, while we consider a harmonic oscillator. More importantly, in the approach used in Ref. [24], in order to study the noise spectrum for high frequencies it is necessary to introduce pulse sequences which, if properly designed, allow for the suppression of the effects of the low frequency parts of the spectrum. In this respect, our approach is simpler, since in order to measure different frequency regions of the noise spectrum, one needs simply to change the trap's frequency.
Experimental scheme.-As we can see in Eq. (13), the rate by which the harmonic oscillator will heat depends strongly upon its mechanical frequency $\omega_{m}$ and the strength of the noise source in the region around that frequency. By taking account of the initial phonon number $n_{0}$ and the effects of other baths coupled to the system, this can be used to probe the spectrum of the noise induced upon the system by the bath of interest $\mathcal{B}$. The effectiveness of such a probe will be determined by the following factors.

(i) The range over which its mechanical frequency $\omega_{m}$ can be adjusted.

(ii) The lower limit of the temperature in which the oscillator can be prepared, with twofold purpose. Firstly, the colder the oscillator, the more accurate the approximation made above in which the bath has significantly higher energy than the oscillator, allowing us to ignore damping effects; and secondly, the lower the value of $\left\langle\hat{n}_{0}\right\rangle$, the lower its spread, and the more accurately $\Delta \hat{n}$ can be deduced.

(iii) The accuracy with which the other baths not being measured can be estimated. In particular, the approximation that they are Markovian (and hence possess a flat spectrum) must be reasonable.

(iv) The accuracy with which $\langle\hat{n}\rangle_{t}$ can be measured.

(v) The ability to increase or decrease the coupling to the bath of interest $\mathcal{B}$. This is not strictly necessary, but as shown in Ref. [25], such a capability is essential in certain contexts for distinguishing between the effects of interest and the effects of other baths.

(vi) The duration of the experiment-as shown in the previous section, the longer we can let the system get heated by the bath, the higher the accuracy of the spectrum measurement.

We claim that an experiment built around a charged levitated nanosphere is well suited to these needs. A "hybrid-type" trap, composed of a quadrupole electric field trap working in conjunction with an optical trap as described in Refs. [25,26], is ideal: it has an exceptionally low noise floor, it is capable of cooling the particle to a very low occupation (in principle, to the ground state) using the techniques outlined in Ref. [27], and the mechanical frequency can be changed at will over the impressively wide range from $\sim 100$ to $\sim 10^{6} \mathrm{~Hz}$. The Paul trap used to levitate the nanosphere is structurally the same as those used as ion traps: an oscillating electric field holds a charged particle in a harmonic well with variable frequency.

The theoretical modeling of noise sources affecting the levitated sphere is a well-trodden path [25,28-32]. Full details of the specific baths treated are available in Appendix 3 of the Supplemental Material [23], but for the purpose of the following section, we will combine them into a single Markovian bath possessing the two-time correlation function $C(t, s)=\delta(t-s) D_{p}$. We take a high-temperature approximation for this Markovian bath, allowing us to neglect any damping effects it might induce. 
Then this bath adds to Eq. (13) as a linear term $D_{p}^{\prime} t$. The mechanical frequency is given by $[26,33]$

$$
\omega_{m}=\frac{V_{0} \beta Q}{\sqrt{2} m \Omega_{d} d^{2}},
$$

where $Q$ is the number of elementary charges attached to the nanosphere, $\beta$ is a form factor of the trap geometry, and $V_{0}$ is the amplitude of the ac voltage applied to the electrodes - the dc component being set to 0 . The mass of the nanosphere is given by $m, d$ gives the distance to the end cap electrodes, and $\Omega_{d}$ is the driving frequency of the trap. The range of $\omega_{m}$ which can be reliably tested over is constrained by the stability of the trap [34], as well as by physical restrictions on various parameters.

Probing the spectrum of electric field noise.-The system described above takes generality as one of its main strengths - it could be used to analyze any noise source to which it could be coupled to, and over a wide frequency range. By way of example, we now demonstrate how such a system could be used for a particular real-world application, which could be achieved with current technology.

The specific structure of the electric field noise which affects the levitated particles in Paul traps remains unknown [12]. Here we demonstrate how our protocol could be utilized to reconstruct it. A simpler but less realistic example, where a noise with a purely Gaussian spectrum is considered, is given in Appendix 4 of the Supplemental Material [23]. Taking achievable parameters for experimental factors (such as background gas pressure, electric field noise, and environment temperature), and using an example of a $R=50 \mathrm{~nm}$ sphere with the density of silica $\rho=2300 \mathrm{~kg} / \mathrm{m}^{3}$ and a charge of $Q=10^{3} e$, we can expect a heating rate from conventional sources of $D_{p}^{\prime} \leq$ 100 phonon $/ \mathrm{s}$ at frequencies higher than $10^{3} \mathrm{~Hz}$.

The basic formulation for the heating rate due to electric field fluctuations in terms of phonons per second is given by $[12]$

$$
D_{E}^{\prime}\left(\omega_{m}\right)=\frac{Q^{2}}{4 m \omega_{m}} S_{E}\left(\omega_{m}\right),
$$

in which $S_{E}\left(\omega_{m}\right)$ is the spectral density of the $E$ field noise at the mechanical frequency of the oscillator. In Ref. [12], by appraising and comparing a wide range of electrically levitated experiments, Brownnutt et al. attain the following general form for this spectral density:

$$
S_{E}(\omega)=g_{E} \omega^{-\alpha} d^{-\beta} T^{\gamma},
$$

in which $g_{E}$ is a scaling constant, $2 d$ is the interelectrode distance, and $T$ is the temperature of the electrodes. $\alpha, \beta$, and $\gamma$ are parameters which depend upon trap geometry and experimental specifics. Here, rather than taking $\alpha$ to be a constant, we will replace it with a structure of arbitrary complexity to make $S_{E}(\omega) \propto \tilde{C}(\omega)$. Accordingly, our phonon expectation value is given by

$$
\begin{aligned}
\langle\hat{n}\rangle_{t}= & \langle\hat{n}\rangle_{0}+D_{p}^{\prime} t \\
& +\frac{k_{E}}{2 \pi m \omega_{m}} \int_{-\infty}^{+\infty} d \nu \tilde{C}(\nu) \frac{\sin ^{2}\left[\left(\omega_{m}-\nu\right) t / 2\right]}{\left(\omega_{m}-\nu\right)^{2}},
\end{aligned}
$$

in which $D_{p}^{\prime}$ gives the effects of all heating sources other than the electric field noise (which, again, are taken as Markovian and without damping), and

$$
k_{E}=Q^{2} d^{-\beta} T^{\gamma} g_{E} .
$$

Now, by selecting an appropriate value for $g_{E}$, we can interrogate how well an arbitrary $\tilde{C}(\nu)$ could be reconstructed in our setting, noting that Eq. (14) would need to be updated to

$$
\tilde{C}\left(\omega_{m}\right)=\frac{4 m \omega_{m}}{k_{E} t}\left(\langle n\rangle_{t}-\langle n\rangle_{0}-D_{p}^{\prime} t\right)
$$

with the term $D_{p}^{\prime} t$ accounting for the Markovian bath. Note that $\tilde{C}\left(\omega_{m}\right)$ depends on $\omega_{m}$ also through $\langle n\rangle_{t}$, which captures the structure of the spectrum of the noise.

Figure 1 demonstrates the capabilities of the technique for recovering a hypothetical complicated structure assigned to the electric field noise (this structure is represented by the dashed blue line). The solid colored lines show what the experimentalist would reconstruct via the spectrometer, using Eq. (20), from the measured values of $\langle n\rangle_{t}$ as a function of $\omega_{m}$. The figure gives a clear demonstration of how the resolution changes with the measurement time. The shortest measurement time - that of the purple line-recovers only the features at highest frequencies, and with poor fidelity; whereas the long measurement time of the green line gives a faithful reconstruction of the objective function. An additional interesting feature is the ringing effect seen at $\omega_{m}>10^{5} \mathrm{~Hz}$ for the purple line. This ringing effect occurs when there are features in the noise structure sharper than the accuracy $\sim 1 / t$ used for reconstructing the spectrum. On the contrary, if the resolution used is high enough, such a ringing is absent. As such, this ringing forms a useful component of the tool. If the experimentalist collects data and infers a particular noise structure from their data, they may wonder if there are finer-grained structures which they might have recovered with longer measurement times. The presence or absence of the characteristic ringing serves to answer the question: if it is there, then the noise contains further features to be discovered. We explain this further in Appendix 5 of the Supplemental Material [23].

The practicability of increasing measurement times inversely proportional to the mechanical frequency makes the technique increasingly viable at higher frequencies; at these high frequencies the accuracy of the reconstruction 


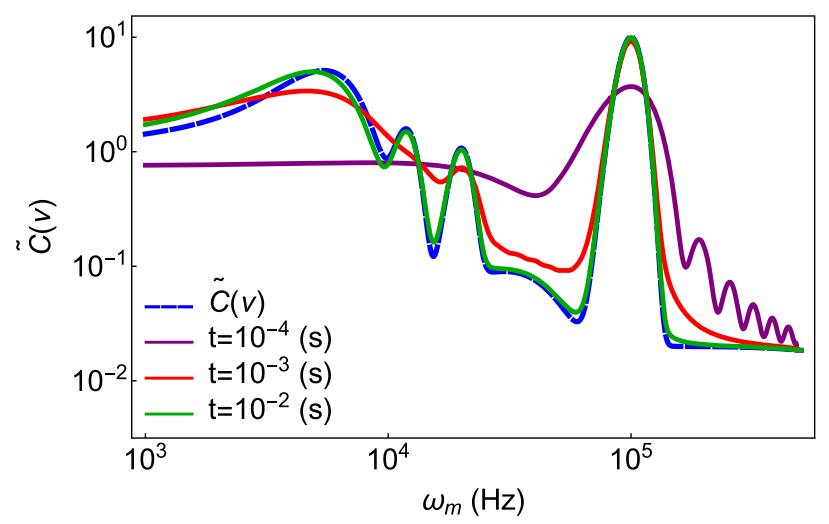

FIG. 1. Simulated performance of the spectrometer as enacted on a levitated nanosphere system with realistic parameters, deployed to measure a fictional and nontrivial structure to the electric field noise. The dashed blue line gives the noise spectrum to be measured, $\tilde{C}(\nu)$. An ideal spectrometer would capture this perfectly. The three solid lines show what an experimenter using our protocol would reconstruct, with the purple, red, and green lines showing reconstructions using measurement times of $t=10^{-4} \mathrm{~s}, t=10^{-3} \mathrm{~s}$, and $t=10^{-2} \mathrm{~s}$, respectively. The longer measurement times allow for a higher fidelity reconstruction of the objective function-in particular, the features at lower frequencies. The parameters utilized for the model are achievable with present-day technology, and the scaling of the $E$ field noise has been chosen such that even with the noise density structure shown it would yield heating rates commensurate with those typically reported in the literature. Simulation parameters and the theoretical treatment of the noise sources can be found in Appendix 3 of the Supplemental Material [23].

will be limited more by the sensitivity of the readout and the preparation of the initial state than by the allowed time for evolution. We also note that the electric field noise is particularly distinguishable in this context due to its $Q^{2}$ scaling, which can be used to disambiguate between the effects of the bath of interest and those of other baths.

A further example connected to non-Markovian models of spontaneous wave function collapse [35-40] is presented in Appendix 2 of the Supplemental Material [23], which also contains Refs. [41-57].

Conclusion.-It is our submission that such a method communicated here would have a broad applicability. Whether for the noise fields invoked by collapse theories, or for more generic non-Markovian quantum noises, this method may be used to detect and characterize the fields of interest. This may be of use in a wide range of technologies, including quantum sensing and improving quantum computing architectures. Further, such a detection and characterization would shine a clear light for theorists trying to determine the physical origins of such fields-since the specific characteristics of a field will carry signatures of its genesis.

We wish to thank Professor H. Ulbricht, Professor M. Paternostro, Dr. Brandon Rodenburg, Dr. A. Smirne, and J. Haase for their valuable suggestions and helpful discussions on this work. D.G. thanks the Centre for Doctoral Training in Controlled Quantum Dynamics. P. B. acknowledges support from the H2020 FET TEQ (Grant No. 766900). S. D. acknowledges support from Fondazione Angelo Della Riccia, the Foundation BLANCEFLOR Boncompagni Ludovisi, née Bildt, the Center for Integrated Quantum Science and Technology (IQST), the Frankfurt Institute for Advanced Studies (FIAS), and the Fetzer Franklin Fund. A. B. acknowledges support from the H2020 FET TEQ (Grant No. 766900), INFN, and the COST action QTSpace.

[1] C. Gardiner and P. Zoller, Quantum Noise (Springer, New York, 2004).

[2] A. A. Clerk, F. Marquardt, and J. G. E. Harris, Phys. Rev. Lett. 104, 213603 (2010).

[3] V. Giovannetti, S. Lloyd, and L. Maccone, Phys. Rev. Lett. 96, 010401 (2006).

[4] H.-P. Breuer, E.-M. Laine, J. Piilo, and B. Vacchini, Rev. Mod. Phys. 88, 021002 (2016).

[5] A. W. Chin, S. F. Huelga, and M. B. Plenio, Phys. Rev. Lett. 109, 233601 (2012).

[6] A. Rivas, S. F. Huelga, and M. B. Plenio, Rep. Prog. Phys. 77, 094001 (2014).

[7] V. Frey, S. Mavadia, L. Norris, W. De Ferranti, D. Lucarelli, L. Viola, and M. Biercuk, Nat. Commun. 8, 2189 (2017).

[8] L. M. Norris, D. Lucarelli, V. M. Frey, S. Mavadia, M. J. Biercuk, and L. Viola, Phys. Rev. A 98, 032315 (2018).

[9] G. A. Paz-Silva, L. M. Norris, and L. Viola, Phys. Rev. A 95, 022121 (2017).

[10] V. Giovannetti, S. Lloyd, and L. Maccone, Nat. Photonics 5, 222 (2011).

[11] A. Safavi-Naini, E. Kim, P. F. Weck, P. Rabl, and H. R. Sadeghpour, Phys. Rev. A 87, 023421 (2013).

[12] M. Brownnutt, M. Kumph, P. Rabl, and R. Blatt, Rev. Mod. Phys. 87, 1419 (2015).

[13] A. Safavi-Naini, P. Rabl, P. F. Weck, and H. R. Sadeghpour, Phys. Rev. A 84, 023412 (2011).

[14] L. Viola, E. Knill, and S. Lloyd, Phys. Rev. Lett. 82, 2417 (1999).

[15] D. Hempston, J. Vovrosh, M. Toroš, G. Winstone, M. Rashid, and H. Ulbricht, Appl. Phys. Lett. 111, 133111 (2017).

[16] G. Winstone, M. Rademacher, R. Bennett, S. Buhmann, and H. Ulbricht, Phys. Rev. A 98, 053831 (2018).

[17] A. O. Caldeira and A. J. Leggett, Physica (Amsterdam) 121A, 587 (1983).

[18] B. L. Hu, J. P. Paz, and Y. Zhang, Phys. Rev. D 45, 2843 (1992).

[19] L. Ferialdi, Phys. Rev. A 95, 052109 (2017).

[20] M. Carlesso and A. Bassi, Phys. Rev. A 95, 052119 (2017).

[21] Y. Sung, F. Beaudoin, L. M. Norris, F. Yan, D. K. Kim, J. Y. Qiu, U. von Lüepke, J. L. Yoder, T. P. Orlando, L. Viola et al., Nat. Commun. 10, 3715 (2019).

[22] L. M. Norris, G. A. Paz-Silva, and L. Viola, Phys. Rev. Lett. 116, 150503 (2016). 
[23] See Supplemental Material at http://link.aps.org/ supplemental/10.1103/PhysRevLett.123.230801 for an alternative derivation under different assumptions, as well as an analysis of likely noise sources in the case of the levitated nanosphere, and a discussion of how the spectrometer could be used to probe collapse models with non-white noises.

[24] Ł. Cywiński, R. M. Lutchyn, C. P. Nave, and S. DasSarma, Phys. Rev. B 77, 174509 (2008).

[25] D. Goldwater, M. Paternostro, and P. F. Barker, Phys. Rev. A 94, 010104 (2016).

[26] J. Millen, P.Z. G. Fonseca, T. Mavrogordatos, T. S. Monteiro, and P. F. Barker, Phys. Rev. Lett. 114, 123602 (2015).

[27] D. Goldwater, B. Stickler, L. Martinetz, T. E. Northup, K. Hornberger, and J. Millen, Quantum Sci. Technol. 4, 024003 (2018).

[28] D. E. Chang, C. A. Regal, S. B. Papp, D. J. Wilson, J. Ye, O. J. Painter, H. J. Kimble, and P. Zoller, Proc. Natl. Acad. Sci. U.S.A. 107, 1005 (2010).

[29] B. Rodenburg, L. Neukirch, A. Vamivakas, and M. Bhattacharya, Optica 3, 318 (2016).

[30] O. Romero-Isart, Phys. Rev. A 84, 052121 (2011).

[31] P. F. Barker and M. N. Shneider, Phys. Rev. A 81, 023826 (2010).

[32] G. A. T. Pender, P. F. Barker, F. Marquardt, J. Millen, and T. S. Monteiro, Phys. Rev. A 85, 021802(R) (2012).

[33] P. Z. G. Fonseca, E. B. Aranas, J. Millen, T. S. Monteiro, and P. F. Barker, Phys. Rev. Lett. 117, 173602 (2016).

[34] W. Paul, Rev. Mod. Phys. 62, 531 (1990).

[35] S. L. Adler and A. Bassi, J. Phys. A 40, 15083 (2007).

[36] S. L. Adler and A. Bassi, J. Phys. A 41, 395308 (2008).

[37] S. L. Adler, A. Bassi, and S. Donadi, J. Phys. A 46, 245304 (2013).

[38] A. Bassi and S. Donadi, Phys. Lett. A 378, 761 (2014).

[39] S. Donadi, D.-A. Deckert, and A. Bassi, Ann. Phys. (Amsterdam) 340, 70 (2014).
[40] M. Carlesso, L. Ferialdi, and A. Bassi, Eur. Phys. J. D 72, 159 (2018).

[41] H.-P. Breuer, F. Petruccione et al., The Theory of Open Quantum Systems (Oxford University Press on Demand, New York, 2002).

[42] A. Bassi, K. Lochan, S. Satin, T. P. Singh, and H. Ulbricht, Rev. Mod. Phys. 85, 471 (2013).

[43] S. L. Adler, A. Bassi, M. Carlesso, and A. Vinante, Phys. Rev. D 99, 103001 (2019).

[44] Q. Fu, Phys. Rev. A 56, 1806 (1997).

[45] K. Piscicchia, A. Bassi, C. Curceanu, R. D. Grande, S. Donadi, B. C. Hiesmayr, and A. Pichler, Entropy 19, 319 (2017).

[46] A. Smirne and A. Bassi, Sci. Rep. 5, 12518 (2015).

[47] J. Nobakht, M. Carlesso, S. Donadi, M. Paternostro, and A. Bassi, Phys. Rev. A 98, 042109 (2018).

[48] M. Bilardello, S. Donadi, A. Vinante, and A. Bassi, Physica (Amsterdam) 462A, 764 (2016).

[49] S. Nimmrichter, K. Hornberger, and K. Hammerer, Phys. Rev. Lett. 113, 020405 (2014).

[50] A. Vinante, R. Mezzena, P. Falferi, M. Carlesso, and A. Bassi, Phys. Rev. Lett. 119, 110401 (2017).

[51] K. Adamiak, IEEE Trans. Ind. Appl. 38, 1001 (2002).

[52] I. Cermák, E. Grün, and J. Švestka, Adv. Space Res. 15, 59 (1995).

[53] P. Asenbaum, S. Kuhn, S. Nimmrichter, U. Sezer, and M. Arndt, Nat. Commun. 4, 2743 (2013).

[54] O. Romero-Isart, M. L. Juan, R. Quidant, and J. I. Cirac, New J. Phys. 12, 033015 (2010).

[55] M. Brownnutt, M. Kumph, P. Rabl, and R. Blatt, Rev. Mod. Phys. 87, 1419 (2014).

[56] Q. a. Turchette, B. E. King, D. Leibfried, D. M. Meekhof, C. J. Myatt, M. A. Rowe, C. A. Sackett, C. S. Wood, W. M. Itano, C. Monroe, and D. J. Wineland, Phys. Rev. A 61, 063418 (2000).

[57] H.-P. Breuer, B. Kappler, and F. Petruccione, Phys. Rev. A 59, 1633 (1999). 